\title{
Switched Feedback Control for a class of First-order Nonholonomic Driftless Systems
}

\author{
Masato Ishikawa* \\ * Kyoto University, Gokasho, Uji, Kyoto 611-0011, JAPAN \\ (e-mail: masato@i.kyoto-u.ac.jp)
}

\begin{abstract}
This paper is concerned with stabilizing feedback control for a class of nonholonomic driftless systems, whose controllability Lie algebra rank condition is satisfied by up to firstorder Lie brackets. We propose a switched feedback law which drives all the initial states to the origin with bounded control inputs (as opposed unbounded, division-by-zero-type discontinuous control). The discontinuity of the feedback law takes place on a subspace defined by the 'parallelism' condition for the base and the fiber vectors in $\mathbb{R}^{3}$ (or simply $\boldsymbol{q} \times \boldsymbol{\phi}=0$ ). We also show that the complement of this discontinuity region is homotopic to $S O(3)$ which is also isomorphic to $\mathbb{S}^{2} \times \mathbb{S}$. The proposed control law is examined by numerical simulations.
\end{abstract}

\section{INTRODUCTION}

In the last two decades, control of nonholonomic driftless systems has been an attractive issue of nonlinear control theory. This is partially because a lot of important mechanical systems with nonintegrable kinematic constraints, such as non-slip rolling constraints or conservation of angular momentum, are modeled as driftless systems. Another reason is that driftless systems cannot be asymptotically stabilized by any continuous state feedback according to Brockett's necessary condition (Brockett [1983]), that motivates us to pursuit non-standard control tools such as discontinuous or time-varying (periodic) feedback control.

Among the subclasses of driftless systems, intensive works have been contributed for chained form systems (Murray et al. [1994]) and their feedback equivalents (Pomet [1992], M.Sampei et al. [1995]). By virtue of the fact that their controllability Lie algebra have particularly simple structure (constructed by iteration of Lie brackets with a special vector-field, called generator), there are wide variety of controllers proposed for this class; in essence, the clue to stabilization problem has been already established.

On the other hand, this paper focuses on a class of driftless systems with 3 inputs and 6 states, whose controllability Lie algebra rank condition is satisfied by up to first-order Lie brackets. We call them first-order systems according to (Murray et al. [1994]), though they are also called differently (Khaneja and Brockett [1999]). Attitude control of 3-D spacecraft using shape changes (Sreenath [1992]) is known as a typical example of first-order systems. The author also proposed a new actual example of this class, called trident snake robot (Ishikawa [2004]), which is a wheeled planar mobile robot with three snake-like branches.

First-order systems are never feedback equivalent to conventional chained systems since there is a structural difference between them. Although the number of studies is relatively small, there have been several challenges to feedback control for non-chained structure systems, such as time-varying feedback control(Khaneja and Brockett [1999]), step-by-step feedback algorithm(Bloch et al. [2000], Iwatani et al. [2002]);

In this paper, we suggest a simple discontinuous feedback control law for this class of systems which brings all the initial states to the origin. We emphasize that this is a complete static state feedback law, in the sense that (i) there is no exception of the control law in which the control input is not assigned; (ii) neither 'step counter' or 'time variable' is used in the control law on the contrary to the existing method. Moreover, the discontinuity of the control law is of sliding-mode type switching, so it is bounded and safer than division-by-zero-type discontinuity (as shown by Tsuchiya et al. [2002]).

This paper is organized as follows. In section 2, we prepare basic notations and the system model. The proposed control law is given in section 3.1 followed by the proofs to show that all the initial states in the state space are brought to the origin in finite time. In section 4 , we also discuss geometric interpretation of the control law and its possible variations. The proposed control law is examined by numerical simulations in section 5 .

\section{PRELIMINARIES}

For a pair of arbitrary spatial vectors $\boldsymbol{a}, \boldsymbol{b} \in \mathbb{R}^{3}, \times$ denotes their cross product defined by

$$
\boldsymbol{a} \times \boldsymbol{b}=\left[\begin{array}{l}
a_{2} b_{3}-a_{3} b_{2} \\
a_{3} b_{1}-a_{1} b_{3} \\
a_{1} b_{2}-a_{2} b_{1}
\end{array}\right]
$$

In the rest of paper, we frequently make use of the formula of scalar triple product:

$$
\boldsymbol{a}^{T}(\boldsymbol{b} \times \boldsymbol{c})=\boldsymbol{b}^{T}(\boldsymbol{c} \times \boldsymbol{a})=\boldsymbol{c}^{T}(\boldsymbol{a} \times \boldsymbol{b})
$$

For a pair of subsets $X$ and $Y, X \backslash Y:=\{x \mid x \in$ $X, x \notin Y\}$ denotes the set subtraction. $X \cong Y$ denotes homeomorphism. 
For a natural number $k, S O(k)$ denotes the $k$-dimensional special orthogonal group and $\mathbb{S}^{k}$ denotes $k$-dimensional unit sphere. $\|\cdot\|$ indicates Euclidean norm.

\subsection{First-order systems with 3 inputs}

Consider the following driftless nonholonomic system

$$
\Sigma_{3}^{6}: \quad \begin{array}{ll}
\dot{\boldsymbol{q}} & =\boldsymbol{u} \\
\dot{\boldsymbol{\phi}} & =\boldsymbol{q} \times \boldsymbol{u}
\end{array}
$$

where $\boldsymbol{q} \in \mathbb{R}^{3}$ is called base vector and $\phi \in \mathbb{R}^{3}$ is called fiber vector. The whole state vector is $\boldsymbol{z}=\left(\boldsymbol{q}^{T}, \boldsymbol{\phi}^{T}\right)^{T} \in \mathbb{R}^{6}$. $\boldsymbol{u} \in \mathbb{R}^{3}$ denotes the control input. Its basic behavior can be interpreted as follows; the base vector $\boldsymbol{q}$ is directly driven by the control input $\boldsymbol{u}$, while $\dot{\boldsymbol{\phi}}$ is given by cross product of the base vector $\boldsymbol{q}$ and its velocity $\dot{\boldsymbol{q}}$, i.e., $\dot{\boldsymbol{\phi}}$ is always perpendicular to the plane including $\boldsymbol{q}$ and $\dot{\boldsymbol{q}}$. Our purpose is to find the control input $\boldsymbol{u}$ which brings the state $\boldsymbol{z}$ from given initial state $\boldsymbol{z}(0)$ to the origin.

If we rewrite the state equation (3) as a vector-field form $\dot{\boldsymbol{z}}=g_{1}(\boldsymbol{z}) u_{1}+g_{2}(\boldsymbol{z}) u_{2}+g_{3}(\boldsymbol{z}) u_{3}$, its controllability distribution is given by

$$
\overline{\mathcal{G}}=C^{\infty} \operatorname{span}\left\{g_{1}, g_{2}, g_{3},\left[g_{1}, g_{2}\right],\left[g_{1}, g_{3}\right],\left[g_{2}, g_{3}\right]\right\}
$$

which has full rank at each point $\boldsymbol{z} \in \mathbb{R}^{6}$. This ensures its local accessibility according to Chow's theorem (see e.g., Nijmeijer and van der Schaft [1990]). Moreover, $\overline{\mathcal{G}}$ is said to be nilpotent of order 2 because all the higher order Lie brackets (such as $\left.\left[g_{1},\left[g_{1}, g_{2}\right]\right]\right)$ are zero. As mentioned above, the 3-D spacecraft with shape controls and the trident snake robot can be modeled as $\Sigma_{3}^{6}$ under nilpotent approximation(see Hermes [1991], Bellaiche et al. [1992], Struemper [1998]).

\section{SWITCHED FEEDBACK CONTROL LAW}

\subsection{Construction of Controlled Invariant Manifolds}

In this section, we propose a switched feedback law for the system $\Sigma_{3}^{6}$. Let us begin with defining an important vector

$$
\boldsymbol{n}:=\boldsymbol{\phi} \times \boldsymbol{q}
$$

which vanishes when $\boldsymbol{q}$ and $\phi$ are parallel. Next, suppose a vector $\boldsymbol{p}$ defined by

$$
\boldsymbol{p}:=\boldsymbol{q} \times \boldsymbol{n},
$$

which is perpendicular to both $\boldsymbol{n}$ and $\boldsymbol{q}$, and belongs to the plane spanned by $\boldsymbol{q}$ and $\boldsymbol{\phi}$ (see Fig. 1 ).

As long as $\boldsymbol{n} \neq 0$, we observe that $(\boldsymbol{q}, \boldsymbol{p}, \boldsymbol{n})$ forms an orthogonal coordinate frame of $\mathbb{R}^{3}$. For any vector $\boldsymbol{x} \in \mathbb{R}^{3}$, let $\boldsymbol{x}_{0}$ denote its normalized vector

$$
\boldsymbol{x}_{0}:=\frac{\boldsymbol{x}}{\|\boldsymbol{x}\|}, \quad \text { if } \boldsymbol{x} \neq 0
$$

where $\|\cdot\|$ indicates Euclidean norm. Note that $\boldsymbol{x}_{0}$ is not defined if $\boldsymbol{x}=0$, while it is bounded and continuous otherwise. Let us define $\boldsymbol{q}_{0}, \boldsymbol{\phi}_{0}, \boldsymbol{n}_{0}$ and $\boldsymbol{p}_{0}$ in this manner.

Now, suppose the following subsets of the state space:

$$
D:=\left\{(\boldsymbol{q}, \boldsymbol{\phi}) \in \mathbb{R}^{6} \mid \boldsymbol{\phi} \times \boldsymbol{q}=0, \boldsymbol{\phi} \neq 0\right\}
$$

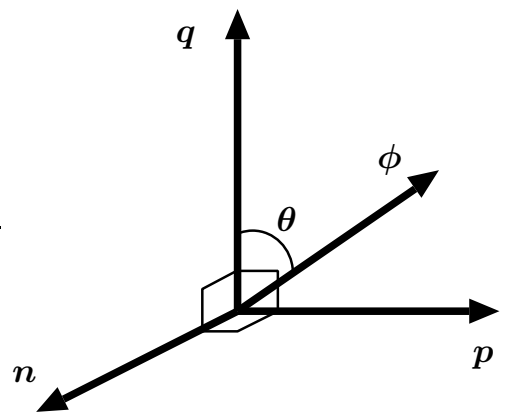

Fig. 1. The base, fiber vectors with their normals in $\mathbb{R}^{3}$

$$
\begin{aligned}
S_{0} & :=\left\{0 \in \mathbb{R}^{6}\right\} \\
S_{1} & :=\left\{(\boldsymbol{q}, \boldsymbol{\phi}) \in \mathbb{R}^{6} \mid \boldsymbol{\phi}=0\right\} \\
S_{2} & :=\left\{(\boldsymbol{q}, \boldsymbol{\phi}) \in \mathbb{R}^{6} \mid \boldsymbol{q}^{T} \boldsymbol{\phi}=0\right\} \cap S_{3} \\
S_{3} & :=\mathbb{R}^{6} \backslash D \\
& =\left\{(\boldsymbol{q}, \boldsymbol{\phi}) \in \mathbb{R}^{6} \mid \boldsymbol{q} \times \boldsymbol{\phi} \neq 0 \text { or } \boldsymbol{\phi}=0\right\}
\end{aligned}
$$

Note that $S_{0} \subset S_{1} \subset S_{2} \subset S_{3} . S_{2}$ is the subset on which $\boldsymbol{q}$ and $\phi$ are orthogonal to each other, while $D$ is the subset on which $\boldsymbol{q}$ and $\boldsymbol{\phi}$ are parallel. Basic idea of our method is to make all $S_{i}$ 's invariant under the proposed control law, and let the trajectory $\boldsymbol{z}$ go through the subsets

$$
D \rightarrow S_{3} \rightarrow S_{2} \rightarrow S_{1} \rightarrow S_{0}
$$

in sequence.

\subsection{Feedback Control Law}

We suggest the following feedback control law

$$
\boldsymbol{u}=\alpha_{q}(\boldsymbol{z}) \boldsymbol{q}_{0}+\alpha_{p}(\boldsymbol{z}) \boldsymbol{p}_{0}+\alpha_{n}(\boldsymbol{z}) \boldsymbol{n}_{0}
$$

where $\alpha_{q}, \alpha_{p}$ and $\alpha_{n}$ are bounded scalar-valued functions chosen as follows:

Case 0: If $z \in S_{0}$ :

$$
\alpha_{q}(\boldsymbol{z})=\alpha_{p}(\boldsymbol{z})=\alpha_{n}(\boldsymbol{z})=0 .
$$

Case 1: If $z \in S_{1}$ :

$$
\begin{aligned}
& \alpha_{q}(\boldsymbol{z})=-k_{q} \\
& \alpha_{p}(\boldsymbol{z})=0 \\
& \alpha_{n}(\boldsymbol{z})=0
\end{aligned}
$$

Case 2: If $z \in S_{2}$ :

$$
\begin{aligned}
& \alpha_{q}(\boldsymbol{z})=k_{q} \operatorname{sgn}(\max \{\|\boldsymbol{\phi}\|, \epsilon\}-\|\boldsymbol{q}\|) \\
& \alpha_{p}(\boldsymbol{z})=0 \\
& \alpha_{n}(\boldsymbol{z})=-k_{n}
\end{aligned}
$$

Case 3: If $z \in S_{3}$ :

$$
\begin{aligned}
& \alpha_{q}(\boldsymbol{z})=0 \\
& \alpha_{p}(\boldsymbol{z})=-k_{p} \operatorname{sgn}\left(\frac{\boldsymbol{\phi}^{T} \boldsymbol{q}}{\boldsymbol{\phi}^{T} \boldsymbol{p}_{0}}\right) \\
& \alpha_{n}(\boldsymbol{z})=0
\end{aligned}
$$

Case 4: If $z \in D$ :

$$
\boldsymbol{u}=\boldsymbol{c}_{0}
$$

where $\boldsymbol{c}_{0} \in \mathbb{R}^{3}$ is any vector satisfying $\boldsymbol{\phi} \times \boldsymbol{c}_{0} \neq 0,\left\|\boldsymbol{c}_{0}\right\|=$ 1.

$k_{q}, k_{n}, k_{p}$ and $\epsilon$ are positive constant design parameters. 


\subsection{Finite-time Reaching to the Origin}

Now we are ready to show that the proposed control law achieves finite-time reaching to the origin for every initial state.

Lemma 1. $S_{1}, S_{2}$ and $S_{3}$ are all invariant under the proposed control law. Moreover, if $\boldsymbol{z}(0)$ belongs to $S_{i+1} \backslash S_{i}$ for some $i \in\{0,1,2\}$, then $\boldsymbol{z}(t)$ will reach $S_{i}$ in finite time.

Proof:

Since $\boldsymbol{q}^{T} \boldsymbol{p}_{0}=0$ and $\boldsymbol{q}^{T} \boldsymbol{n}_{0}=0$, we have

$$
\begin{aligned}
\frac{d}{d t}\|\boldsymbol{q}\| & =\frac{\boldsymbol{q}^{T} \dot{\boldsymbol{q}}}{\|\boldsymbol{q}\|} \\
& =\alpha_{q}(\boldsymbol{z}) \frac{\boldsymbol{q}^{T} \boldsymbol{q}_{0}}{\|\boldsymbol{q}\|} \\
& =\alpha_{q}(\boldsymbol{z})
\end{aligned}
$$

Similarly, since $\boldsymbol{n}^{T} \boldsymbol{q}_{0}=0$ and $\boldsymbol{n}^{T} \boldsymbol{p}_{0}=0$, we have

$$
\begin{aligned}
\frac{d}{d t}\|\boldsymbol{\phi}\| & =\frac{\boldsymbol{\phi}^{T} \dot{\boldsymbol{\phi}}}{\|\boldsymbol{\phi}\|} \\
& =\frac{\boldsymbol{\phi}^{T}(\boldsymbol{q} \times \boldsymbol{u})}{\|\boldsymbol{\phi}\|} \\
& =\frac{\boldsymbol{u}^{T}(\boldsymbol{\phi} \times \boldsymbol{q})}{\|\boldsymbol{\phi}\|} \\
& =\alpha_{n}(\boldsymbol{z}) \frac{\boldsymbol{n}_{0}^{T} \boldsymbol{n}}{\|\boldsymbol{\phi}\|} \\
& =\alpha_{n}(\boldsymbol{z})\|\boldsymbol{q}\| \sin |\theta|
\end{aligned}
$$

where $|\theta|>0$ is the angle between $\boldsymbol{q}$ and $\phi$ (we do not care its sign).

$$
\begin{aligned}
\frac{d}{d t}\left(\boldsymbol{\phi}^{T} \boldsymbol{q}\right) & =\boldsymbol{q}^{T} \dot{\boldsymbol{\phi}}+\boldsymbol{\phi}^{T} \dot{\boldsymbol{q}} \\
& =\boldsymbol{q}^{T}(\boldsymbol{q} \times \boldsymbol{u})+\boldsymbol{\phi}^{T} \boldsymbol{u} \\
& =\boldsymbol{\phi}^{T}\left(\alpha_{q}(\boldsymbol{z}) \boldsymbol{q}_{0}+\alpha_{p}(\boldsymbol{z}) \boldsymbol{p}_{0}\right) \\
& =\alpha_{q}(\boldsymbol{z})\left(\boldsymbol{\phi}^{T} \boldsymbol{q}_{0}\right)+\alpha_{p}(\boldsymbol{z})\left(\boldsymbol{\phi}^{T} \boldsymbol{p}_{0}\right)
\end{aligned}
$$

Here we used $\boldsymbol{q}^{T}(\boldsymbol{q} \times \boldsymbol{u})=\boldsymbol{u}^{T}(\boldsymbol{q} \times \boldsymbol{q})=0$.

Case 0: It is obvious that $S_{0}$ is invariant under $\boldsymbol{u}=0$ (i.e., the origin is an equilibrium of the closed-loop system).

Case 1: From (16) and (25), $\frac{d}{d t}\|\phi\|=0$ is satisfied on $S_{1}$ since $\alpha_{n}(\boldsymbol{z})=0$. This implies $S_{1}$ is invariant.

Moreover, since

$$
\frac{d}{d t}\|\boldsymbol{q}\|=-k_{q}
$$

$\|\boldsymbol{q}(t)\|$ vanishes at $t=\|\boldsymbol{q}(0)\| / k_{q}$. Thus $\boldsymbol{z}(t)$ reaches $S_{0}$ in finite time.

\section{Case 2:}

(Proof of invariance) From the definition (10), $\boldsymbol{q}^{T} \boldsymbol{\phi}=$ 0 is satisfied on $S_{2}$. Moreover, the points $\{\boldsymbol{q}=0, \boldsymbol{\phi} \neq 0\}$ are excluded from $S_{2}$ because they belongs to $D$; i.e., either $\boldsymbol{q} \neq 0$ or $\boldsymbol{\phi}=0$ is satisfied. This leads us to notice that

$$
\boldsymbol{\phi}^{T} \boldsymbol{q}_{0}=\frac{\boldsymbol{\phi}^{T} \boldsymbol{q}}{\|\boldsymbol{q}\|}=0
$$

is satisfied on $S_{2}$. Substituting (17)-(19) into (26), we have

$$
\frac{d}{d t}\left(\phi^{T} \boldsymbol{q}\right)=-\alpha_{q}(\boldsymbol{z})\left(\boldsymbol{\phi}^{T} \boldsymbol{q}_{0}\right)=0
$$

which concludes $S_{2}$ is invariant.

(Proof of finite-time reaching) Next, suppose $\boldsymbol{z}(0) \in$ $S_{2} \backslash S_{1}$, i.e,

$$
\|\boldsymbol{q}(0)\| \neq 0,\|\phi(0)\| \neq 0, \phi(0)^{T} \boldsymbol{q}(0)=0
$$

Let us show that $\|\boldsymbol{q}\|$ reaches $\max \{\|\phi\|, \epsilon\}$ in finite time. If $\|\boldsymbol{q}(0)\|<\max \{\|\boldsymbol{\phi}\|, \epsilon\}$

$$
\frac{d}{d t}\|\boldsymbol{q}\|=k_{q}
$$

until $\|\boldsymbol{\phi}\|-\|\boldsymbol{q}\|=0$. Thus $\|\boldsymbol{q}\|$ is increasing, and

$$
\|\boldsymbol{q}(t)\|=\|\boldsymbol{q}(0)\|+k_{q} t .
$$

On the other hand, substituting (19) into (25) and considering $\sin |\theta|=1$, we have

$$
\begin{aligned}
\frac{d}{d t}\|\boldsymbol{\phi}\| & =-k_{n}\|\boldsymbol{q}\| \\
& =-k_{n}\left(\|\boldsymbol{q}(0)\|+k_{q} t\right) \\
\|\boldsymbol{\phi}(t)\| & =\|\boldsymbol{\phi}(0)\|-k_{n}\|\boldsymbol{q}(0)\| t-\frac{1}{2} k_{n} k_{q} t^{2}
\end{aligned}
$$

So $\|\phi(t)\|-\|\boldsymbol{q}(t)\|=0$ is a quadratic equation with respect to $t$. It is easy to see that there exists a positive root, say $r_{1}$. Therefore $\|\boldsymbol{q}\|=\max \{\|\boldsymbol{\phi}\|, \epsilon\}$ is satisfied at the time

$$
t=t_{1}, \quad t_{1}:=\max \left\{r_{1}, \frac{\epsilon-\|\boldsymbol{q}(0)\|}{k_{q}}\right\}
$$

It is also true in the case of $\|\phi(0)\|<\|\boldsymbol{q}(0)\|$ by repeating similar argument.

Once $\left\|\boldsymbol{q}\left(t_{1}\right)\right\|=\max \left\{\left\|\boldsymbol{\phi}\left(t_{1}\right)\right\|, \epsilon\right\}$ is satisfied, $\|\boldsymbol{q}(t)\| \geq$ $\epsilon$ will hold afterward. Thus we obtain

$$
\begin{aligned}
& \frac{d}{d t}\|\phi\| \leq-k_{n} \epsilon \\
& \|\phi(t)\| \leq\left\|\phi\left(t_{1}\right)\right\|-k_{n} \epsilon\left(t-t_{1}\right),
\end{aligned}
$$

so it is clear that $\|\phi(t)\|$ reaches $\mathbf{0}$ no later than

$$
t=t_{2}, \quad t_{2}:=t_{1}+\frac{\left\|\phi\left(t_{1}\right)\right\|}{k_{n} \epsilon},
$$

i.e., $\boldsymbol{z}(t)$ reaches $S_{1}$ in finite time.

Case 3: In order to prove $S_{3}=\mathbb{R}^{6} \backslash D$ is invariant, it is sufficient to show that any trajectory starting from $S_{3}$ will not get close to $D$. Substituting (20)-(22) into (26), we have

$$
\begin{aligned}
\frac{d}{d t}\|\boldsymbol{q}\| & =0 \\
\frac{d}{d t}\|\boldsymbol{\phi}\| & =0 \\
\frac{d}{d t}\left(\boldsymbol{\phi}^{T} \boldsymbol{q}\right) & =-k_{p} \operatorname{sgn}\left(\frac{\boldsymbol{\phi}^{T} \boldsymbol{q}}{\boldsymbol{\phi}^{T} \boldsymbol{p}_{0}}\right)\left(\boldsymbol{\phi}^{T} \boldsymbol{p}_{0}\right) \\
& =-k_{p}\left|\boldsymbol{\phi}^{T} \boldsymbol{p}_{0}\right| \operatorname{sgn}\left(\boldsymbol{\phi}^{T} \boldsymbol{q}\right)
\end{aligned}
$$

Therefore both $\|\boldsymbol{q}\|$ and $\|\boldsymbol{\phi}\|$ are kept constant, while $\left|\phi^{T} \boldsymbol{q}\right|$ is strictly decreasing. This implies $|\cos \theta|$ is decreasing, thus $\boldsymbol{z}$ will not approach $D$.

Note that $\boldsymbol{z}(0) \in S_{3} \backslash S_{2}$ implies $\|\boldsymbol{q}(0)\| \neq 0$ and $\|\phi(0)\| \neq 0$. Since

$$
\frac{d}{d t}\left(\boldsymbol{q}^{T} \boldsymbol{\phi}\right)=k_{p} \boldsymbol{q}_{0}^{T} \boldsymbol{\phi}=-\|\boldsymbol{\phi}\| \operatorname{sgn}\left(\boldsymbol{q}^{T} \boldsymbol{\phi}\right)
$$


we can see that $\boldsymbol{q}^{T} \boldsymbol{\phi}$ vanishes in finite time, i.e., $\boldsymbol{z}(t)$ reaches $S_{2}$ in finite time.

Lemma 2. No trajectory stays on $D$.

Proof:

Since Case 4 of the control law is chosen when $\boldsymbol{z} \in D$, let us compute the derivative of $\boldsymbol{n}$ under (23).

$$
\begin{aligned}
\frac{d}{d t} \boldsymbol{n} & =\dot{\boldsymbol{q}} \times \boldsymbol{\phi}+\boldsymbol{q} \times \dot{\boldsymbol{\phi}} \\
& =-\boldsymbol{\phi} \times \boldsymbol{c}_{0}+\boldsymbol{q} \times\left(\boldsymbol{q} \times \boldsymbol{c}_{0}\right) \\
& =-\boldsymbol{\phi} \times \boldsymbol{c}_{0}+\kappa^{2} \boldsymbol{\phi} \times\left(\boldsymbol{\phi} \times \boldsymbol{c}_{0}\right)
\end{aligned}
$$

where we introduced $\kappa \in \mathbb{R}$ such that $\boldsymbol{q}=\kappa \boldsymbol{\phi}$ because $\boldsymbol{q}$ is parallel to $\phi$. Considering $\phi \times \boldsymbol{c}_{0} \neq 0$ and $\left(\boldsymbol{\phi} \times \boldsymbol{c}_{0}\right) \perp(\phi \times$ $\left.\left(\phi \times c_{0}\right)\right)$, we can conclude that $\frac{d}{d t} \boldsymbol{n} \neq 0$ on $D$. This implies $\dot{\boldsymbol{z}}$ does not belong to the tangent space of $D$. Every trajectory starting from $D$ will leave $D$ (thus enter $S_{3}$ ), in infinitesimally short time.

The observations above are summarized into the following theorem.

Theorem 3. Under the proposed control law (12), any trajectory $\boldsymbol{z}(t)$ starting from $\mathbb{R}^{6}$ reach the origin in finite time.

The proof is omitted because it is direct combination of Lemma 1 and Lemma 2. Let us close this section with summarizing the effect of each terms in the control law (12):

- The term $\alpha_{q}(\boldsymbol{z}) \boldsymbol{q}_{0}$ contributes to control of $\|\boldsymbol{q}\|$.

- The term $\alpha_{n}(\boldsymbol{z}) \boldsymbol{n}_{0}$ contributes to control of $\|\phi\|$; basically this effect corresponds to the principle of holonomy.

- The term $\alpha_{p}(\boldsymbol{z}) \boldsymbol{p}_{0}$ is used to change the angle between $\phi$ and $\boldsymbol{q}$.

\section{DISCUSSION}

\subsection{Underlying topology of the control law}

The proposed method can be regarded as a natural extension of the sliding-mode controller proposed by Bloch and Drakunov [1996], for Brockett integrator

$$
\begin{array}{ll}
\Sigma_{2}^{3}: & \dot{\boldsymbol{q}}=\boldsymbol{u} \\
\dot{\phi}=q_{2} u_{1}-q_{1} u_{2}
\end{array}
$$

where $\boldsymbol{q} \in \mathbb{R}^{2}, \phi \in \mathbb{R}$ and $\boldsymbol{z}:=\left(\boldsymbol{q}^{T}, \phi\right)^{T} \in \mathbb{R}^{3}$. They suggested a control law

$$
\boldsymbol{u}=-\alpha_{q}(\boldsymbol{z}) \boldsymbol{q}-\alpha_{n}(\boldsymbol{z})\left[\begin{array}{c}
-q_{2} \\
q_{1}
\end{array}\right]
$$

which has exception at $D:=\{\boldsymbol{q}=0, \phi \neq 0\}$; i.e., all the trajectory starting from $\mathbb{R}^{3} \backslash D$ moves towards the origin $\{0\}$. A topological interpretation of the effect of introducing this $D$ is as follows. If there had been a continuous state feedback, it would have had non-zero value on $\mathbb{R}^{3} \backslash\{0\}$. Now, from the viewpoint of homotopy equivalence, $\mathbb{R}^{3} \backslash\{0\}$ is homotopic to $\mathbb{S}^{2}$. Namely, the support (complement of the kernel) of the control law would have been homotopic to $\mathbb{S}^{2}$. On the other hand, once the set of exception $D$ is introduced, we consider that the whole state space is restricted to $\mathbb{R}^{3} \backslash D$. Then the support of the control law becomes $\left(\mathbb{R}^{3} \backslash D\right) \backslash\{0\}$, which is homotopic to $S O(2) \cong \mathbb{S}^{1}$. Roughly speaking, the introduction of $D$ is a topological operation which remodels $\mathbb{S}^{2}$ into $\mathbb{S}^{1}$ (indeed, this is the way to get rid of Brockett's necessary condition; see Coron [1990] for topological generalization of Brockett's condition).

Let us turn to apply this interpretation to $\Sigma_{3}^{6} . \mathbb{R}^{6} \backslash D$ is equal to $S_{3}$ as we defined in (11), so $\left(\mathbb{R}^{6} \backslash D\right) \backslash\{0\}$ is equal to $S_{3} \backslash\{0\}$.

Theorem 4. $S_{3} \backslash\{0\}$ is homotopic to $S O(3)$.

Proof:

To each point $\boldsymbol{z} \in S_{3} \backslash\{0\}$, assign the $3 \times 3$-matrix defined by

$$
G(z):=[\boldsymbol{q}, \boldsymbol{\phi}, \boldsymbol{\phi} \times \boldsymbol{q}] .
$$

Since all the column vectors of $G(\boldsymbol{z})$ is linearly independent on $S_{3} \backslash\{0\}$, we see that $\operatorname{det} G$ is sign definite, namely, $G$ provides one-to-one correspondence between $S_{3} \backslash\{0\}$ and $G L_{+}(3)$ (the set of $3 \times 3$-matrix whose determinant is positive, which is clearly homotopic to $S O(3))$. From this correspondence, we can conclude that $S_{3} \backslash\{0\}$ is homotopic to $S O(3)$.

Note that $S O(3)$ is homeomorphic to $\mathbb{S}^{2} \times \mathbb{S}^{1}$. If there had been a continuous state feedback, it would have had nonzero value on $\mathbb{R}^{6} \backslash\{0\}$, which is homotopic to $\mathbb{S}^{5}$. Therefore, we can say that the topological effect of introducing $D$ is to remodel $\mathbb{S}^{5}$ into $S O(3) \simeq \mathbb{S}^{2} \times \mathbb{S}^{1}$. A rough visualization of this observation is depicted in Fig. 2. In the case of $\Sigma_{2}^{3}$, the support of the control law is homotopic to $\mathbb{S}^{1}$, which is just a 'circle'. While in the case of $\Sigma_{3}^{6}$, it is $\mathbb{S}^{2} \times \mathbb{S}^{1}-$ a circle is assigned to every point of a sphere.

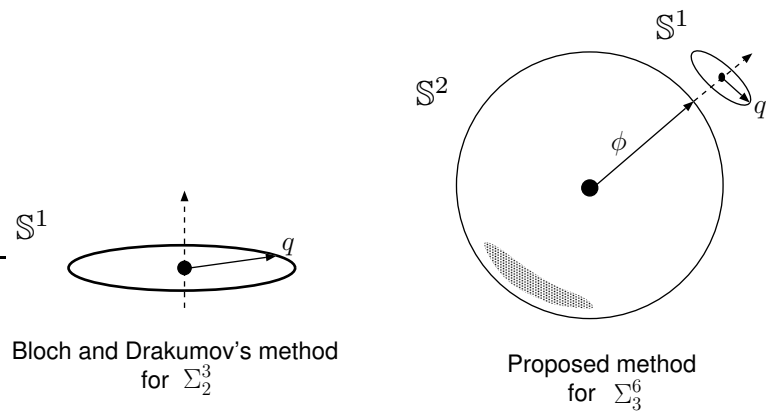

Fig. 2. Topological image of the proposed control law

\subsection{Partial continuation of the control law}

The feedback control law proposed in Section 3.1 is discontinuous (as a function of state vector $\boldsymbol{z}$ ) at each point of $D$, as well as on $S_{1}, S_{2}$ and $S_{3}$. However, the meaning of discontinuity is different.

Discontinuity of the control law at the points of $D$ is caused by the nature of $\boldsymbol{n}_{0}=\boldsymbol{n} /\|\boldsymbol{n}\|$. Suppose a point $\boldsymbol{z}^{*} \in D$. Then $\boldsymbol{n}_{0}$ is not defined at $\boldsymbol{z}^{*}$ because $\boldsymbol{n}$ vanishes there, while

$$
\lim _{\boldsymbol{z} \rightarrow \boldsymbol{z}^{*}} \boldsymbol{n}_{0}
$$

converges to a constant vector, depending on the direction in which $z$ approaches to $z^{*}$. Thus this discontinuity is of 
signum-type referring to $x /|x|=\operatorname{sgn} x$ in scalar case. Let us say it essential discontinuity.

On the other hand, the discontinuity of $\alpha_{p}(\boldsymbol{z})$, which is found in (21) defined on $S_{3}$, is not essential; it is practically avoidable, e.g., by simply replacing the signum function by standard sigmoid function (see Fig.3).

$$
\sigma(x)=\frac{1-\exp \left(-k_{\sigma} x\right)}{1+\exp \left(-k_{\sigma} x\right)}, \quad k_{\sigma}>0
$$
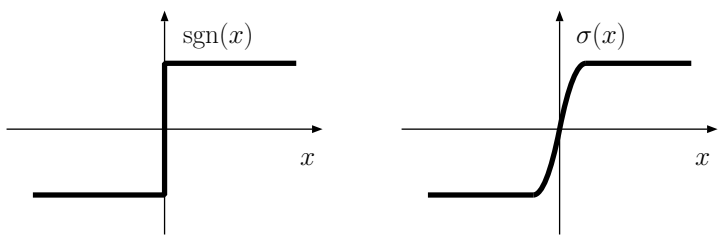

Fig. 3. Signum and Sigmoid functions

Considering the discontinuity of $\alpha_{q}(\boldsymbol{z})$ and $\alpha_{n}(\boldsymbol{z})$ on $S_{1}, S_{2}$ recalls exactly the same argument discussed by Bloch and Drakunov [1996], since the behavior of the system $\Sigma_{3}^{6}$ restricted to $S_{2}$ is essentially the same as that of system $\Sigma_{2}^{3}$. For example, other choices for $\alpha_{q}(\boldsymbol{z})$ are possible in (17), such as

$$
\begin{aligned}
\alpha_{q}(\boldsymbol{z}) & =k_{q} \operatorname{sgn}(\|\boldsymbol{\phi}\|-\|\boldsymbol{q}\|), \\
\text { or } \quad \alpha_{q}(\boldsymbol{z}) & =k_{q} \operatorname{sgn}(\epsilon-\|\boldsymbol{q}\|),
\end{aligned}
$$

each of which will yield different convergence results (see the aforementioned literature for detail). In any case, we should remember that there is a trade-off between the speed of convergence/reaching and smoothness of the control input.

\section{SIMULATION}

Let us examine the proposed method by numerical simulations. Figures (4)-(8) show the simulation results for

$$
\boldsymbol{z}(0)=(\boldsymbol{q}(0), \boldsymbol{\phi}(0))=(-0.05,0.1,-0.1,5,-1,1)^{T}
$$

as initial condition. Note that $\boldsymbol{z}(0)$ belongs to $D$ in this case because $\boldsymbol{q}(0)$ and $\phi(0)$ are parallel. For smoothness of the numerical computation, the signum functions are blurred by substituting sigmoid function with $k_{\sigma}=1.0 \times$ $10^{3}$. Other design parameters are chosen as $k_{q}=k_{p}=$ $k_{n}=1, \epsilon=0.5$.

Fig. 4 and 5 show the time history of the state vectors $\boldsymbol{q}$ and $\phi$, while Fig. 6 shows the corresponding control input $\boldsymbol{u}$. At the beginning of the simulation, $\boldsymbol{z}$ instantaneously exits from $D$ driven by Case 4 of the control law, and enters $S_{3}$. Then Case 3 of the control law is chosen, so that $\phi$ and $\boldsymbol{q}$ become orthogonal to each other in finite time. Both $\|\boldsymbol{q}\|$ and $\|\boldsymbol{\phi}\|$ are kept constant (see Fig.7) while $\boldsymbol{\phi}^{T} \boldsymbol{q}$ decreases and $\|\boldsymbol{n}\|$ increases (see Fig.8). Right after $\boldsymbol{z}(t)$ reaches $S_{2}$ at around $t=0.25$, Case 2 of the control law is chosen in turn. Since the radius $\|\boldsymbol{q}\|$ is smaller than the required level $\|\phi\|$ at this moment, it increases until $\|\boldsymbol{q}\|$ becomes equal to $\max \{\|\phi\|, \epsilon\}$; it turns to decrease after that. Meanwhile, $\|\phi\|$ decreases monotonically to $\mathbf{0}$ by virtue of the holonomy term $-k_{n} \boldsymbol{n}_{0} \cdot \boldsymbol{z}(t)$ reaches $S_{1}$ at around $t=2.8[\mathrm{sec}]$. Finally, $\boldsymbol{q}$ goes directory to $\mathbf{0}$ driven by Case 1 of the control law.
In addition, Fig. 9 shows the corresponding trajectory of $\boldsymbol{q}$ and $\boldsymbol{\phi}$ plotted in $\mathbb{R}^{3}$, to help the readers' geometrical comprehension. We can see that $\phi$ goes almost straightly to the origin, while $\boldsymbol{q}$ leaves a circle-like trajectory (which is mainly the trace of Case 2).

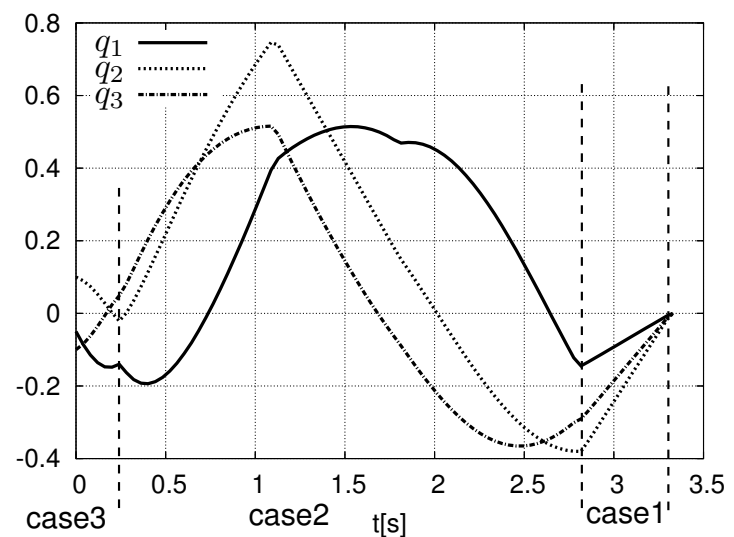

Fig. 4. Time history of $\boldsymbol{q}$

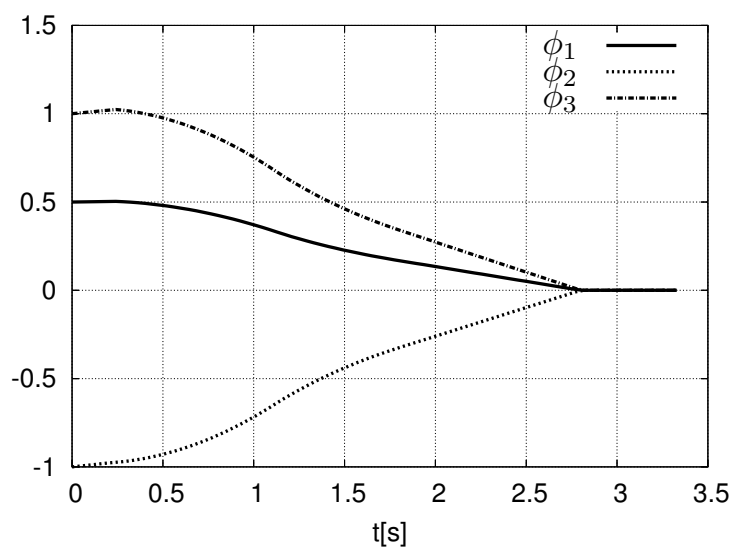

Fig. 5. Time history of $\phi$

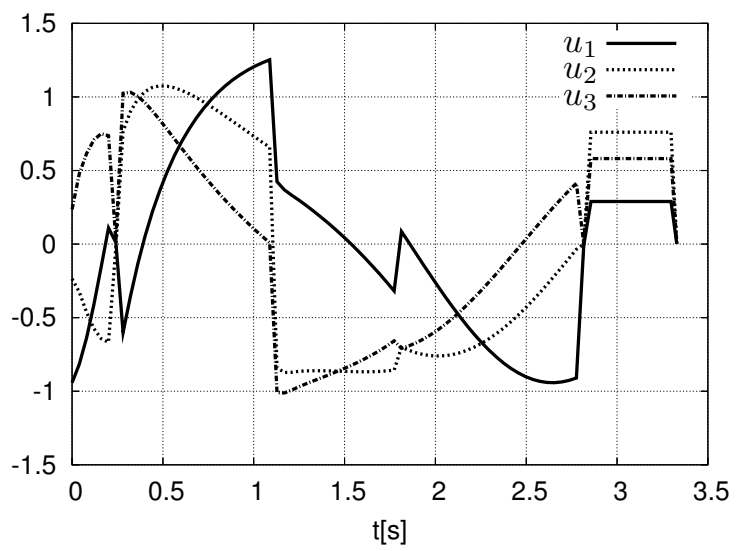

Fig. 6. The control input $\boldsymbol{u}$

\section{CONCLUSION}

In this paper, we suggested a switched feedback control law for first-order driftless nonholonomic systems with 3inputs. The proposed method guarantees the boundedness of the control inputs and finite-time reaching to the origin 


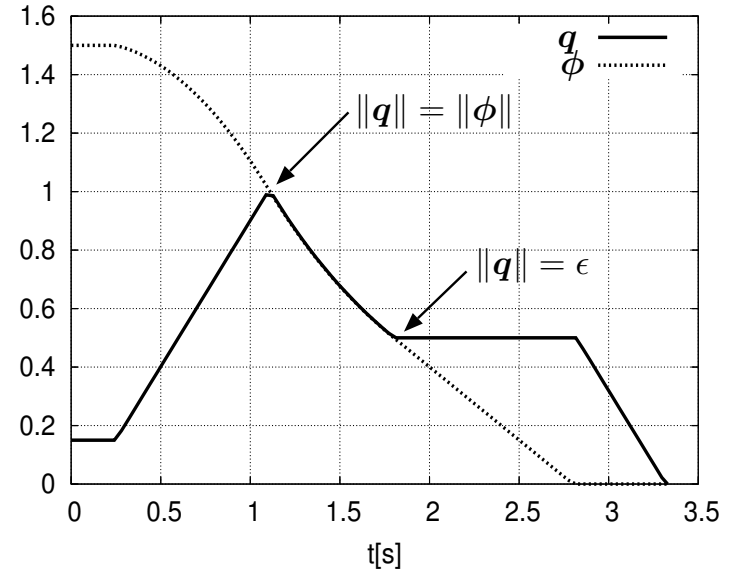

Fig. 7. Time history of $\|\boldsymbol{q}\|,\|\phi\|$

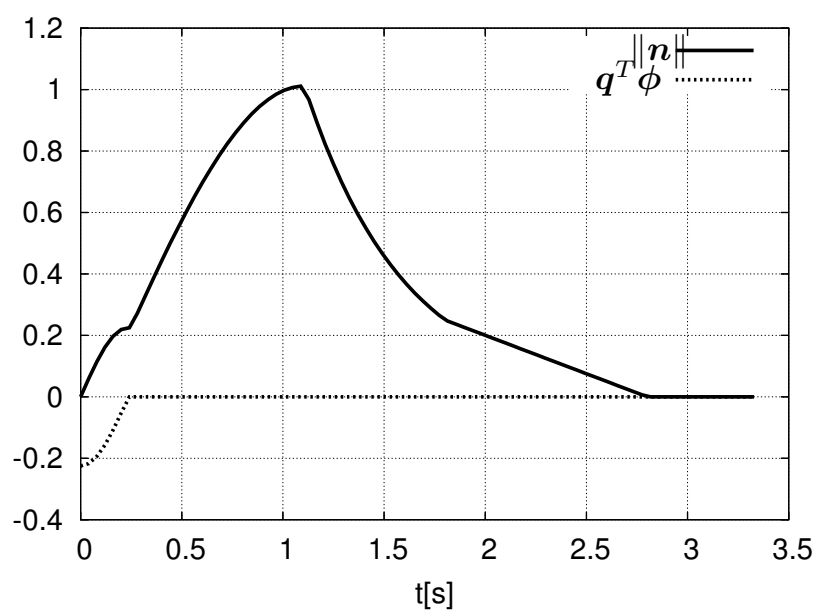

Fig. 8. Time history of $\|\boldsymbol{n}\|, \boldsymbol{\phi}^{T} \boldsymbol{q}$

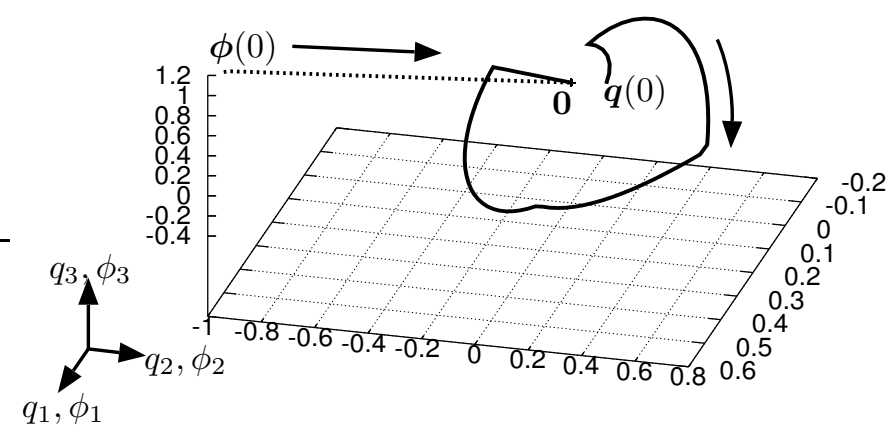

Fig. 9. Spatial trajectory of $\boldsymbol{q}, \boldsymbol{\phi}$ on $\mathbb{R}^{3}$

for all initial states without exception. Its extension to non-nilpotent systems without approximation, such as trident snake robot, will be considered in future works.

\section{ACKNOWLEDGEMENTS}

This study was supported in part by the Grant-in-Aid for Young Scientists (B), No.19760285 of the Ministry of Education, Science, Sports and Culture, Japan, 20072009.

\section{REFERENCES}

A. Bellaiche, J.-P. Laumond, and J.-J. Risler. Nilpotent infinitesimal approximations to control lie algebra. In Proc. IFAC Nonlinear Control Systems Design, pages 101-108, Bordeaux,France, 1992.

A.M. Bloch. Nonholonomic Mechanics and Control, volume 24 of Interdisciplinary Applied Mathematics (IAM). Springer Verlag, 2003.

A.M. Bloch and S.V. Drakunov. Stabilization and tracking in the nonholonomic integrator via sliding modes. Systems \&5 Control Letters, 29:91-99, 1996.

A.M. Bloch, S.V. Drakunov, and M.K. Kinyon. Stabilization of nonholonomic systems using isospectral flows. SIAM Journal on Control and Optimization, 38(3):855$874,2000$.

R.W. Brockett. Asymptotic stability and feedback stabilization. In Differential Geometric Control Theory, volume 27, pages 181-191. Springer Verlag, 1983.

J.M. Coron. A necessary condition for feedback stabilization. Systems \& Control Letters, 14:227-232, 1990.

H. Hermes. Nilpotent and high order approximations of vector fields systems. SIAM Review, 33:238-264, 61991.

M. Ishikawa. Trident snake robot: Locomotion analysis and control. In IFAC NOLCOS, pages 1169-1174, 2004.

Y. Iwatani, M. Ishikawa, and S. Hara. Switched feedback control of first-order symmetric affine systems via timestate control form. In $A C C^{\prime} 02,2002$.

N. Khaneja and R.W. Brockett. Stabilization of brockett's generalized canonical driftless systems. In Proc. of the 38th IEEE Conference on Decision and Control, pages 1640-1645, 1999.

M.Sampei, H.Kiyota, and M.Ishikawa. Time-state control form and its application to a non-holonomic space robot. In Proc. of IFAC NOLCOS'95, pages 759-764, 1995.

R.M. Murray, Z. Li, and S.S. Sastry. A Mathematical Introduction to Robotic Manipulation. CRC Press, 1994.

H. Nijmeijer and A.J. van der Schaft. Nonlinear Dynamical Control Systems. Springer Verlag, 1990.

J.-B. Pomet. Explicit design of time-varying stabilizing control laws for a class of controllable systems without drift. Systems \& Control Letters, 18:147-158, 1992.

N. Sreenath. Nonlinear control of planar multibody systems in shape space. Mathematics of Control, Signals, and Systems, 5(5):343-363, 1992.

H. Struemper. Nilpotent approximation and nilpotentization for under-actuated systems on matrix lie groups. In IEEE Conf. on Decision and Control, pages 4188-4193, 1998.

T. Tsuchiya, T. Urakubo, and K. Tsujita. Motion control of a nonholonomic system based on the lyapunov control method. Journal of Guidance, Control, and Dynamics, $25(2): 285-290,2002$. 